\title{
Proteção e roteamento multicaminho em redes ópticas elásticas com multiplexação por divisão espacial
}

\author{
Helder M. N. da S. Oliveira ${ }^{1}$, Nelson L. S. da Fonseca ${ }^{1}$ \\ ${ }^{1}$ Instituto de Computação - Universidade Estadual de Campinas (UNICAMP) \\ Campinas 13083-852, SP, Brasil \\ helderalrc.ic.unicamp.br, nfonsecalic.unicamp.br
}

\begin{abstract}
The introduction of space division multiplexing in optical networks brought new challenges for network protection since lightpaths can allocate great capacity and transmit data at different rates. In addition, these networks suffer from the fragmentation of the spectrum that hinders contiguity and continuity constraints and, therefore, increases blocking. To address these problems, in this paper, we propose a protection algorithm for elastic optical networks with spatial division multiplexing based on multipath routing and shared backup paths.
\end{abstract}

Resumo. A introdução da multiplexação por divisão espacial em redes ópticas traz novos desafios para a proteção da rede, uma vez que o caminho da luz pode abranger alta capacidade e transmitir dados a taxas diferentes. Além disso, essas redes sofrem com a fragmentação do espectro que dificulta as restrições de contiguidade e continuidade e, portanto, aumentam o bloqueio. Para resolver esses problemas, neste artigo, propomos um algoritmo de proteção para redes ópticas elásticas com multiplexação por divisão espacial baseado em roteamento multicaminho e caminhos de backup compartilhados.

\section{Introdução}

A rede óptica elástica (EON) com multiplexação por divisão espacial (SDM), EON-SDM, é uma solução promissora para lidar com as crescentes demandas de largura de banda. A capacidade de alocar de maneira flexível o espectro permitirá que esse tipo de rede lide com as demandas de tráfego com vários requisitos de largura de banda. Além disso, a adoção da multiplexação por divisão espacial proporcionará maior disponibilidade de capacidade. Quanto maior a capacidade da rede e o tráfego transportado, maior é a necessidade de esquemas de proteção eficazes para evitar a perda maciça de dados. No entanto, o emprego de capacidade de reserva para a proteção de caminhos de trabalho reduz os recursos disponíveis para caminhos de trabalho, levando ao bloqueio de solicitações de entrada.

O estabelecimento e a remoção de caminhos ópticos acarreta na fragmentação do espectro, que é um estado no qual existem slots disponíveis, mas descontinuos de tal forma que não podem ser usados para aceitar novas solicitações, isto é, a segmentação do espectro disponível em pequenas bandas não contíguas. O uso do roteamento multicaminho oferece a vantagem de usar pequenas faixas contíguas divididas em diversos caminhos para a requisição, possibilitando o aumento do número de solicitações aceitas. A Figura 1 ilustra como o roteamento multicaminho possibilita a aceitação de requisições que não seria aceita através de roteamento simples.

Em redes ópticas que utilizam multiplexação por divisão de comprimento de onda (WDM), o uso do roteamento multicaminho destina-se principalmente a rotear 


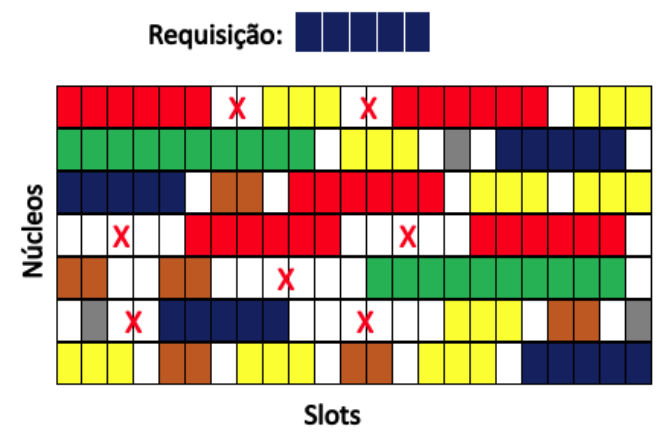

Roteamento Simples
Nova Requisição:

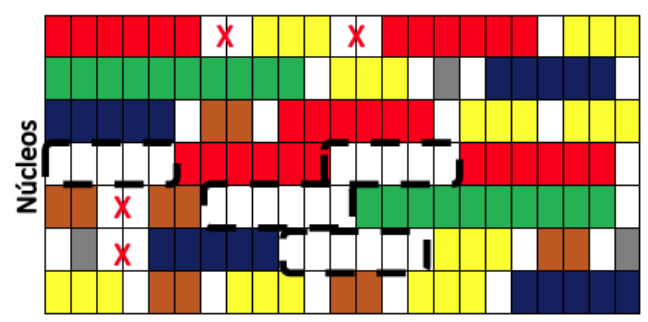

Slots

Roteamento Multicaminho

Figura 1. Fragmentação do espectro

aplicações com grandes requisitos de grande largura de banda, geralmente maiores que a capacidade de um comprimento de onda [Chen et al. 2009b, Chen et al. 2009a, de Santi et al. 2012, de Santi et al. 2015, de Santi and da Fonseca 2014]. No entanto, em redes ópticas elásticas com multiplexação por divisão espacial, o roteamento multicaminho possibilita aumentar a utilização dos recursos da rede. Além da melhor utilização dos recursos, o roteamento multicaminhos em EON-SDM pode gerar menor probabilidade de bloqueio [Ruan and Zheng 2014], e incremento de sobrevivência [Ruan and Zheng 2014], já que o transporte do volume total da demanda ocorre em diversos caminhos.

Segundo [Consortium 2002], duas das características que podem ser utilizadas para determinar um conjunto de caminhos a serem utilizados é o número de caminhos disponíveis entre os nós origem e destino e a independência dos caminhos, ou seja, quão disjuntos são os caminhos que compõem o conjunto de múltiplos caminhos. Este artigo propõe um algoritmo de proteção em EON-SDM que utiliza multicaminho e avalia o desempenho do algoritmo proposto em função do número de caminhos necessário para suprir uma demanda de tráfego.

Apesar de outros trabalhos terem estudado a proteção de redes ópticas elásticas com multiplexação por divisão espacial [Tode and Hirota 2014, Oliveira and da Fonseca 2016, Muhammad et al. 2014], nenhum dos trabalhos na literatura emprega multicaminho para diminuir o bloqueio gerado pela fragmentação do espectro. Este artigo introduz o algoritmo Multipath Protection for Multicore network (MPMN) para diminuir a fragmentação gerada pela alocação e desalocação de caminhos ópticos em redes ópticas elásticas com multiplexação por divisão espacial utilizando multicaminhos. Além disso, o algoritmo MPMN decide sobre caminhos de proteção, utilizando caminhos compartilhados.

Este artigo está organizado da seguinte forma. A seção 2 revisa trabalhos relacionados. A seção 3 introduz o algoritmo MPMN. A seção 4 avalia o desempenho do algoritmo proposto e a seção 5 conclui o artigo.

\section{Trabalhos relacionados}

Apesar de algoritmos de roteamento e alocação de espectro e núcleo terem sido propostos na literatura, poucos artigos consideraram a proteção da rede. Além disso, roteamento multicaminho não foi considerado nesses estudos.

Os autores de [Gao et al. 2016] propuseram uma formulação baseada em 
programação linear inteira (PLI) e uma heurística de roteamento multicaminho e alocação de espectro para redes ópticas elásticas protegidas. O esquema proposto atinge uma menor utilização de recursos em comparação com os esquemas de proteção tradicionais. Além de multicaminho, os algoritmos citados utilizaram diferentes formatos de modulação.

Em [Goścień et al. 2016] estuda-se a sobrevivência de redes ópticas elásticas baseada em roteamento multicaminho, permitindo a transmissão de uma demanda em diferentes rotas, garantindo a transmissão de um volume de demanda específico no caso de uma falha de um único enlace. O artigo propõe uma PLI e uma heurística para resolver o problema de sobrevivência em redes ópticas elásticas utilizando multicaminho.

Chen et.al em [Chen et al. 2013] propuseram dois algoritmos de roteamento multicaminho e de atribuição de espectro utilizando modulação adaptativa de acordo com a distância entre os nós finais para redes ópticas elásticas. Eles analisaram a eficácia dos algoritmos em cenários de tráfego dinâmico. Os autores mostraram que a combinação de roteamento multicaminho e modulação adaptativa em redes ópticas elásticas reduz o bloqueio de requisições em comparação ao uso de roteamento de caminho único.

Em [Dharmaweera et al. 2016] investigou-se o emprego conjunto de agregação de tráfego e multicaminho para redes ópticas elásticas. Através de simulações, os autores demonstraram que a agregação de tráfego e roteamento multicaminho, aumentam a eficiência espectral e reduz o consumo de recursos em relação aos esquemas existentes.

Os autores de [Ruan and Zheng 2014] consideraram o impacto da fragmentação do espectro ao longo de caminhos simples e multicaminhos em redes ópticas elásticas. Demonstraram que o esquema de multicaminhos atinge maior eficiência espectral que o esquema de provisionamento com caminho único.

Apesar de diversos trabalhos terem avaliado o uso de multicaminho para se prover proteção em redes ópticas elásticas, nenhum destes trabalhos trataram de proteção de redes ópticas elásticas com multiplexação por divisão espacial.

\section{O algoritmo MPMN}

O algoritmo introduzido nesta seção, chamado Multipath Protection for Multicore network (MPMN), garante $100 \%$ de proteção para todos os caminhos estabelecidos em uma rede óptica elásticas com multiplexação por divisão espacial. O algoritmo MPMN divide o tráfego por $k$ caminhos para atender a demanda de uma conexão requisitada. Além disso, cada caminho criado possui um caminho de proteção. Os $k$ caminhos são escolhidos considerando a disponibilidade de núcleo e espectro nos enlaces da rede. Neste algoritmo, o caminho primário e o seu caminho de proteção correspondente são totalmente disjuntos, uma vez que a disponibilidade de um deles deve ser garantida em caso de um falha. Contudo, os diferentes caminhos primários e de proteção não necessitam respeitar esta restrição.

Neste artigo, a topologia da rede foi modelada como um grafo $G(V, E, W)$, composto de um conjunto de nós $V$, um conjunto de arestas $E$ e um conjunto de pesos de arestas $W$. As arestas que conectam dois vértices de $G$ representam os enlaces da rede. Uma requisição é denotada como $R(s, d, r)$, onde $s, d \in V$ são os nós de origem e destino, e a largura de banda requirida é $r \mathrm{~Gb} / \mathrm{s}$. O número de intervalos de frequência requeridos $b$ para o pedido $R(f, d, r)$ é derivado a partir de $r$.

Para encontrar $k$ caminhos com a quantidade de slots contínuos e contíguos necessária, foram computadas $k+2$ rotas no grafo $G$, dentre as $k+2$ rotas buscou-se um conjunto de slots que respeitasse a restrição de contiguidade e continuidade. Os $k$ menores caminhos considerando as restrições de continuidade e contiguidade de núcleo e 
espectro são então os caminhos escolhidos. As rotas dos caminhos primários e do caminhos de proteção são encontrados através do algoritmo de Yen[Yen 1970]. Os slots de frequência ao longo desta rota são determinados através do emprego da política first-fit. Se o algoritmo de alocação de espectro retornar com sucesso, os recursos encontrados serão alocados. Caso contrário, o próximo núcleo é selecionado e examinado para o mesmo caminho. As $k+2$ rotas serão testadas para encontrar os $k$ caminhos. Se existir espectro disponível, os recursos encontrados são alocados para a requisição. Se todos os núcleos tiverem sido examinados e o espectro não puder ser encontrado na rota, o próximo caminho candidato será analisado.

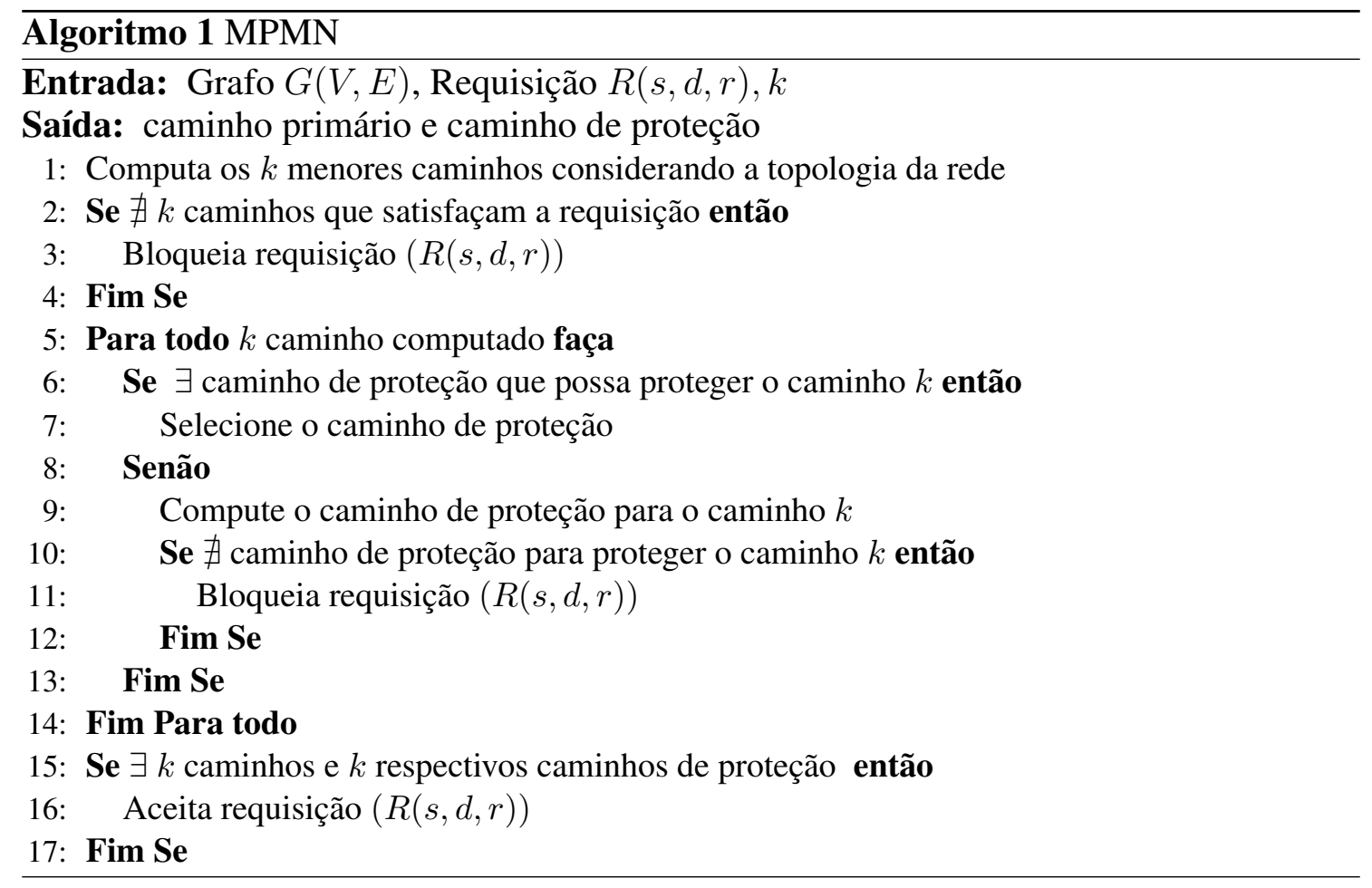

No algoritmo MPMN (Algoritmo 1), a linha 1 busca $k$ caminhos primários. Se não existir $k$ caminhos primários disponíveis (linha 2), a requisição é bloqueada (linha 3). Caso contrário, para cada um dos $k$ caminhos, encontra-se um caminho de proteção (linha 5). Na linha 6 , um caminho de proteção para proteger o $k$-ésimo caminho primário será buscado dentre os caminhos de proteção já estabelecidos para outras requisições. Se não existir nenhum caminho de proteção que possa proteger a requisição, então um novo caminho precisa ser criado (linha 9). Se um caminho de proteção para proteger o $k$-ésimo caminho primário não puder ser encontrado (linha 10), a requisição é bloqueada (linha 11). Se $k$ caminhos primários forem encontrados e todos os caminhos forem protegidos por um caminho de proteção a requisição é aceita (linha 16).

Para encontrar o caminho primário, no pior caso, o algoritmo de Yen, que possui complexidade amortizada de $O(E+V \log V)$, é executado. Da mesma forma, para o caminho de proteção, o algoritmo de Yen é executado. A complexidade de encontrar os slots contínuos e contíguos é $O(E+V)$. Logo, no pior caso, o algoritmo MPMN tem complexidade de $O(E+V \log V)$.

\section{Resultados numéricos}

Para avaliar o desempenho do algoritmo proposto, experimentos de simulação foram realizados utilizando o simulador FlexGridSim [Moura and Drummond ]. Em cada simulação, 
100.000 requisições foram geradas; utilizou-se o método de replicação independente e adotou-se nível de confiança de $95 \%$ para os intervalos de confiança. Diferentes topologias com diferentes conectividades foram empregadas nas simulações, a Pan-Europan (Figura 2.1) com 28 nós e 39 enlaces bidirecionais, a USA (Figura 2.2) com 24 nós e 43 enlaces bidirecionais e a NSF (Figura 2.3) com 14 nós e 20 enlaces bidirecionais. O espectro foi dividido em 320 slots de espectro e as fibras simulada possuem 7 núcleos. A carga variou entre 25 e 500 erlangs em intervalos de 25 erlangs. Sete tipos de requisições foram utilizadas 25 Gbps, 50 Gbps, 125 Gbps, 200 Gbps, 500 Gbps, 750 Gbps e 1 Tbps.

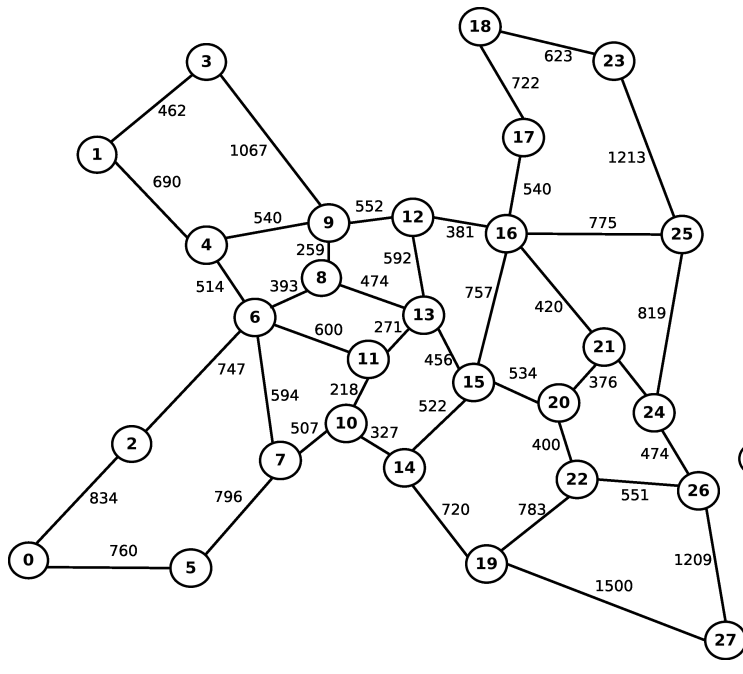

2.1 Pan-European

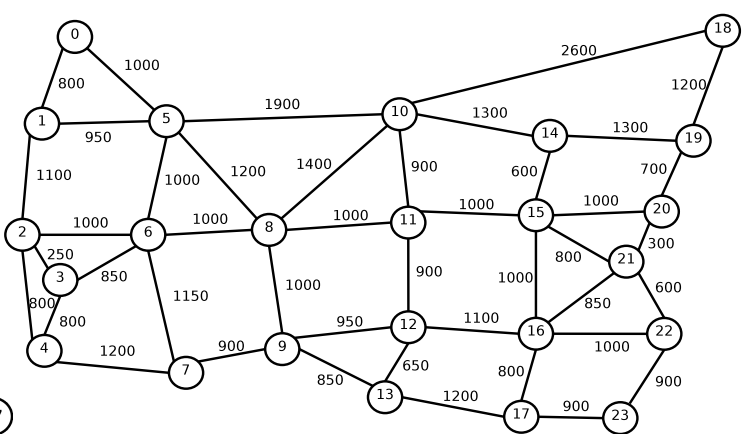

2.2 USA

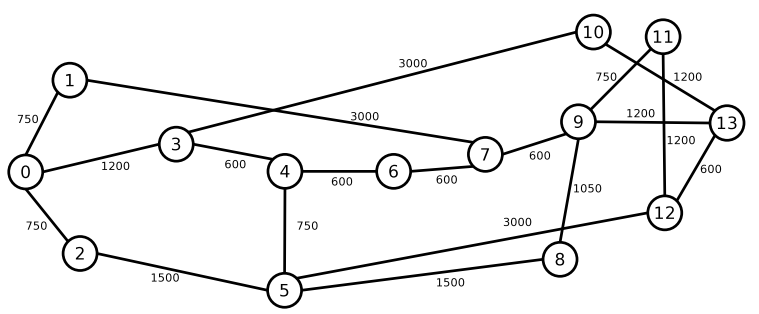

$2.3 \mathrm{NSF}$

Figura 2. Topologias

O algoritmo MPMN foi avaliado em função do número de caminhos necessário para suprir uma demanda de tráfego. Nas figuras, as curvas denotadas por $k=1$ mostram os resultados para redes usando o algoritmo MPMN e roteamento de caminho único, enquanto as curvas denotados por $k=2, k=3$ e $k=4$ mostram, respectivamente, resultados para redes usando o algoritmo MPMN com as requisições divididas em dois, três e quatro multicaminhos. Utilizou-se um limite de cerca de $-32 \mathrm{~dB}$ como aceitável na alocação de um recurso. Para calcular o crosstalk (XT) de um núcleo com $n$ núcleos vizinhos ativos de uma fibra com múltiplos núcleos homogêneos, utilizou-se a Equação 1 [Hayashi et al. 2011, Saridis et al. 2015].

$$
h=\frac{2 \cdot k^{2} \cdot C}{\beta \cdot D}
$$

$$
X T=\frac{n\{1-\exp (-(n+1) \cdot 2 \cdot h \cdot L\}}{1+n\{\exp (-(n+1) \cdot 2 \cdot h \cdot L)\}}
$$

Na Eq. 1, $h$ é o aumento da interferência média por unidade de comprimento, 
calculado por vários parâmetros da fibra: $k, \beta, C, D$, são, respectivamente, o coeficiente de acoplamento, a constante de propagação, o raio de curvatura e a distância entre os núcleos.

A Eq. 2 faz uso de $h$ da equação 1 multiplicada por $L$, que é o comprimento da fibra, enquanto $n$ representa o número de núcleos adjacentes.

Assumiu-se um valor máximo de $n=6$, Além disso, os outros parâmetros assumiram valores realísticos, sendo $k=2 \times 10^{-5}, C=50 \mathrm{~mm}, \beta=4 \times 10^{6}$ e $D=45 \mu \mathrm{m}$. O pior caso de crosstalk para os núcleos sempre será o núcleo central (ou qualquer outro núcleo que tenha o maior número de núcleos vizinhos), uma vez que recebe interferência indesejada de todos os seus núcleos adjacentes. Enfatiza-se que o crosstalk inter-núcleo ocorre apenas entre os mesmos slots de frequência usados em núcleos adjacentes. Nas simulações, para o cálculo do crosstalk assume-se que o espectro de cada núcleo é totalmente utilizado. As métricas consideradas para avaliação dos algoritmos são a probabilidade de bloqueio de banda, o valor de crosstalk por slot, a razão de fragmentação, a eficiência energética, o número de saltos para o caminho primário e o número de saltos para o caminho de proteção.

A probabilidade de bloqueio de banda é definida pela porcentagem de banda (tráfego) bloqueada sobre o total de banda solicitada durante todo o período de simulação. O valor de crosstalk por slot é definido como a relação média entre os pares de slots de frequência usados que têm a mesma frequência e estão localizados em núcleos adjacentes e o total de slots usados. A relação de fragmentação é gerada a partir do estabelecimento e a desconexão de caminhos. A razão de fragmentação compara o número máximo de slots contíguos disponíveis ao número de slots disponíveis no enlace. A medida de eficiência energética é obtida dividindo-se a demanda de tráfego total atendida com sucesso na rede pelo consumo total de energia da rede. O número de saltos do caminho primário apresenta a média do número de saltos de todos caminhos primário estabelecidos por conexão. $\mathrm{O}$ número de saltos do caminho de backup apresenta a média do número de saltos de todos caminhos de backup estabelecidos por conexão.

As Figuras 3.1, 3.2 e 3.3 mostram a probabilidade de bloqueio (BBR) para a topologia Pan-European, USA e NSF, respectivamente. Para a topologia Pan-European (Figura 3.1), o algoritmo MPMN com diferentes valores para $k$ inicia o bloqueio de requisições sob cargas de 75 erlangs. Para cargas entre 75 e 200 erlangs o algoritmo MPMN com caminho único $(k=1)$ produz maior BBR que para os casos de uso de multicaminhos, independentemente do valor de $k$. Para $k=3$ o algoritmo MPMN produz os menores valores de BBR, seguido de $k=2$ e $k=4$, isto ocorre devido a dificuldade de encontrar 4 caminhos quando utilizado $k=4$ e de manter a continuidade para $k=2$. Sob cargas mais altas, o algoritmo MPMN com caminho único produz a menor BBR devido às características da conectividade da rede Pan-European, que dificulta a produção de multicaminhos em determinados nós da rede. As características da topologia Pan-European propiciam a ocorrência de resultados semelhantes para diferentes valores de $k$. Apesar do algoritmo MPMN não utilizar caminhos disjuntos na criação dos caminhos primários, valores maiores de $k$ implicam na sobrecarga de alguns enlaces. Quanto maior for $k$, maior será o uso de recursos, uma vez que ao se dividir requisições em diferentes caminhos, cada caminho utilizará uma banda de guarda. Com o aumento da carga da rede, a diferença de $\mathrm{BBR}$ entre diferentes valores de $k$ diminui.

Para topologia USA (Figura 3.2), o algoritmo MPMN com multicaminho inicia o bloqueio de requisições sob cargas de 175 erlangs. O algoritmo MPMN com caminho único produz a maior BBR, iniciando o bloqueio sob cargas de 150 erlangs. Considerando 


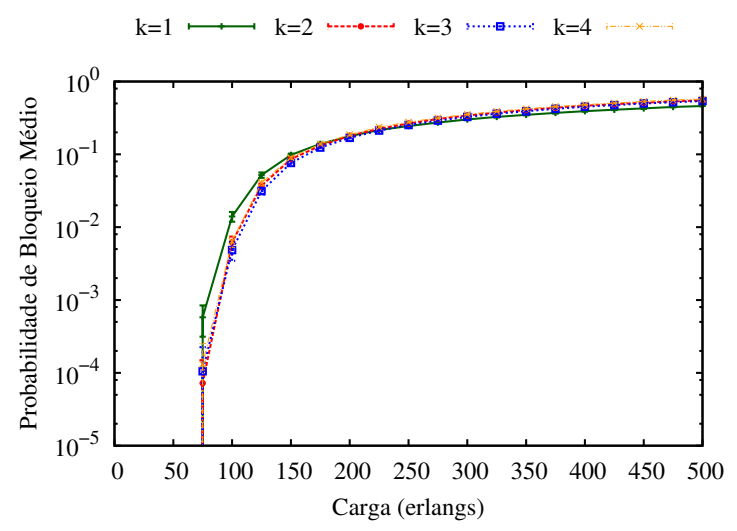

3.1 Pan-European

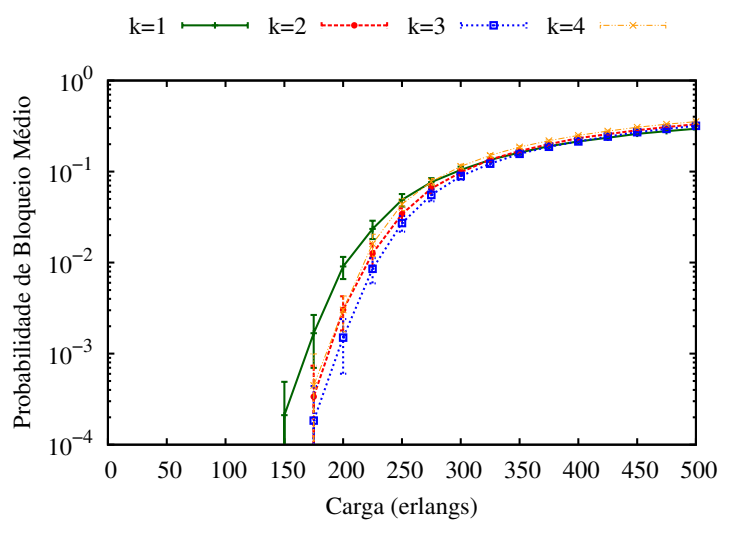

3.2 USA

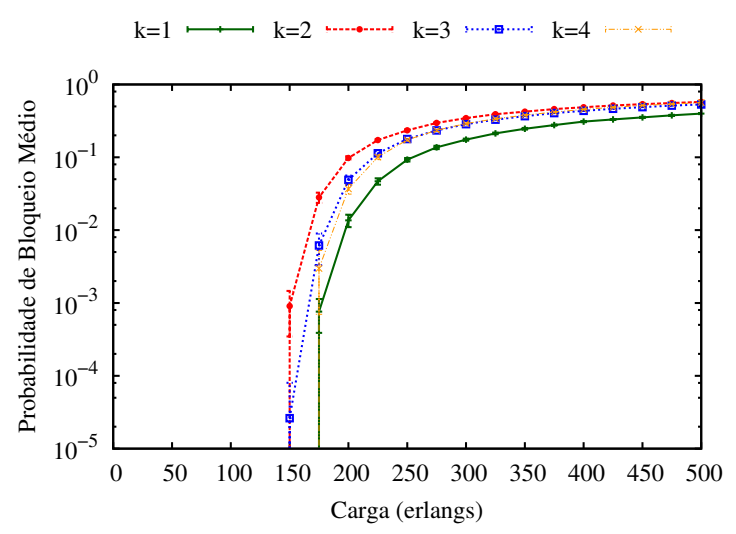

3.3 NSF

Figura 3. Bloqueio de banda em função da carga da rede

diferentes roteamentos multicaminhos, o algoritmo MPMN com $k=3$ produziu a menor BBR. Para esta topologia, o bloqueio entre caminho único e multicaminhos é um pouco mais significativa do que para topologia Pan-European. A alta conectividade desta topologia permite que o algoritmo MPMN com multicaminhos encontre um maior número de caminhos alternativos do que em topologias menos conectadas, o que faz com que os valores de bloqueio para cargas mais altas sejam ainda menores do que as mesmas medidas encontradas para a topologia Pan-European. Sob cargas de 175 erlangs, a utilização de multicaminhos reduz em até uma ordem de grandeza em relação a utilização quando se usa roteamento com caminhos únicos.

Para a topologia NSF (Figura 3.3), o algoritmo MPMN utilizando multicaminhos com $k=2$ e $k=3$ inicia o bloqueio de requisições sob cargas de 150 erlangs, as variações do algoritmo MPMN utilizando caminho único e utilizando multicaminhos com $k=4$ iniciam o bloqueio de requisições sob cargas de 175 erlangs. Diferentemente quando executado nas topologias Pan-European e USA, o algoritmo MPMN utilizando caminho único produz menor BRR do que quando se utiliza multicaminhos para os diferentes valores de $k$; isto acontece devido à baixa conectividade desta topologia o que dificulta o algoritmo MPMN com multicaminhos encontrar $k$ caminhos primários.

As Figuras 4.1, 4.2 e 4.3 mostram a relação de crosstalk por slot $(\mathrm{CpS})$ gerada para as topologias Pan-European, USA e NSF. Para topologia Pan-European (Figura 4.1), os valores de $\mathrm{CpS}$ gerados pelo algoritmo MPMN para $k$ igual a 1, 2, 3 e 4 iniciam com um valor de 0,018 e aumentam até 0,19, 0,15, 0,11 e 0,09, respectivamente. Esses resul- 


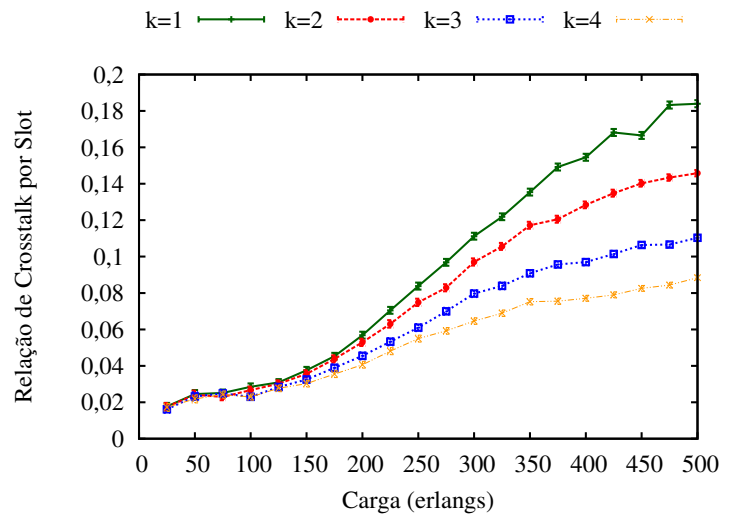

4.1 Pan-European

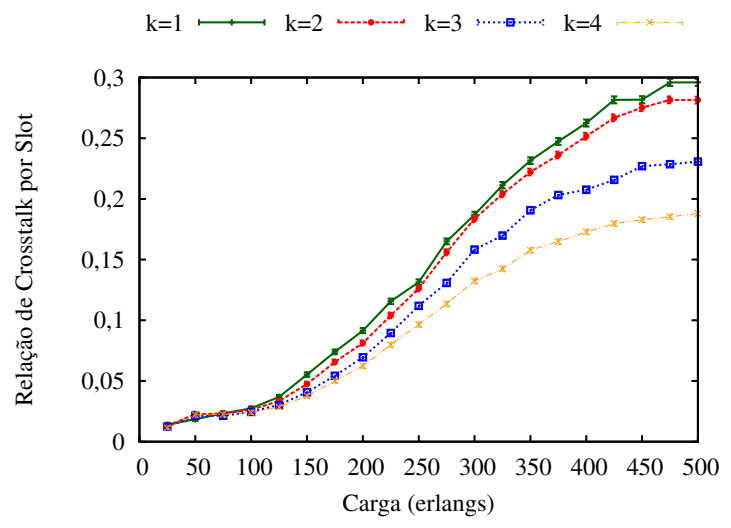

4.2 USA

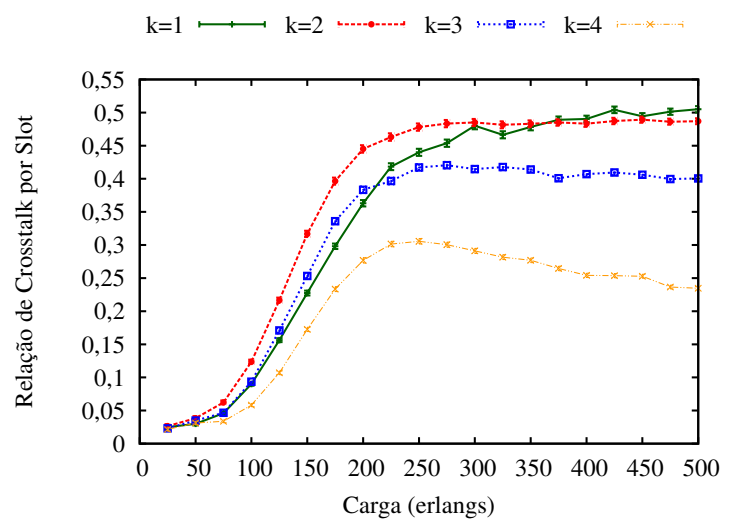

$4.3 \mathrm{NSF}$

Figura 4. Crosstalk por slot em função da carga da rede

tados mostram que para topologia Pan-European a divisão das requisições por múltiplos caminhos melhora o $\mathrm{CpS}$, ou seja, um número menor de slots são afetados pelo crosstalk gerado. Além disso, os bloqueios gerados pelos diferentes valores de $k$ não produzem grande influência nos slots nos quais há crosstalk significativo, uma vez que o algoritmo MPMN com caminho único $(k=1)$ produz a maior BBR e o maior $\mathrm{CpS}$.

Para topologia USA (Figura 4.2), os valores de $\mathrm{CpS}$ gerados pelo o algoritmo MPMN para $k$ igual a 1, 2, 3, 4 iniciam com um valor de 0,011 e aumentam até $0,29,0,28$, 0,23 e 0,18 , respectivamente. Assim como para topologia Pan-European, a utilização de múltiplos caminhos diminui o $\mathrm{CpS}$ gerado, como consequência da maior alocação de recursos na rede. Além disso, assim como para topologia Pan-European, os bloqueios gerados pelos diferentes valores de $k$ não produzem grande influência nos slots que recebem crosstalk, uma vez que o algoritmo MPMN com caminho único produz a maior BBR e o maior CpS. Sob cargas mais altas, quanto maiores forem a carga e o valor de $k$, maior será a diferença entre os valores de $\mathrm{CpS}$ gerados.

Para a topologia NSF (Figura 4.3), o comportamento do CpS gerado por esta topologia difere do $\mathrm{CpS}$ gerado em outras topologias, influenciado pela sua conectividade. O CpS gerado pelo algoritmo MPMN com $k=4$ é bem mais baixo do que o gerado por outros algoritmos. O CpS gerado pelo algoritmo MPMN com caminho único aumenta rapidamente, produzindo baixos valores de $\mathrm{CpS}$ sob baixas cargas e o maior valor de $\mathrm{CpS}$ sob cargas altas. Até 350 erlangs, para $k=2$, produz-se os maiores valores de $\mathrm{CpS}$, influenciado pela média do número médio de saltos do caminho primário. 


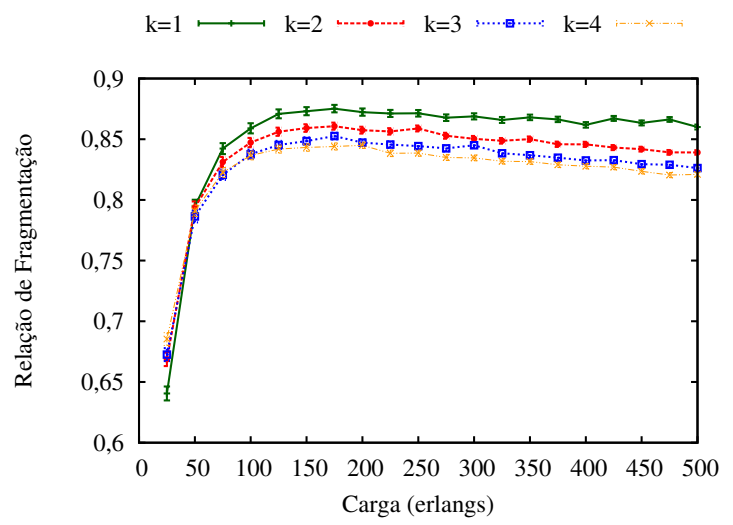

5.1 Pan-European

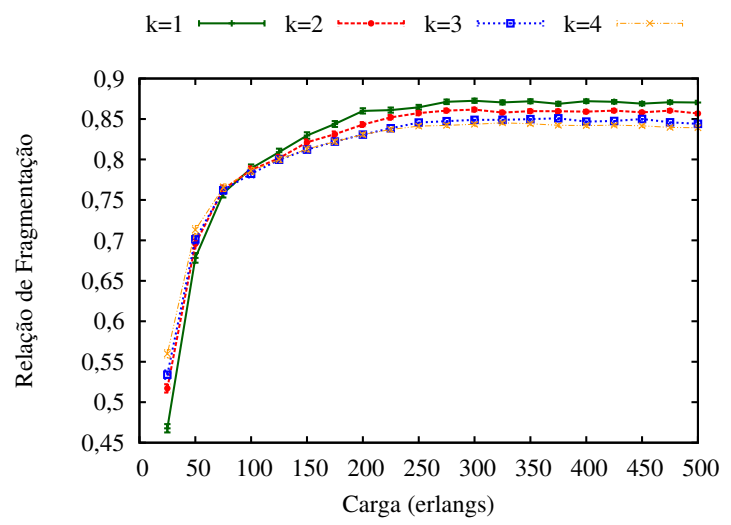

5.2 USA

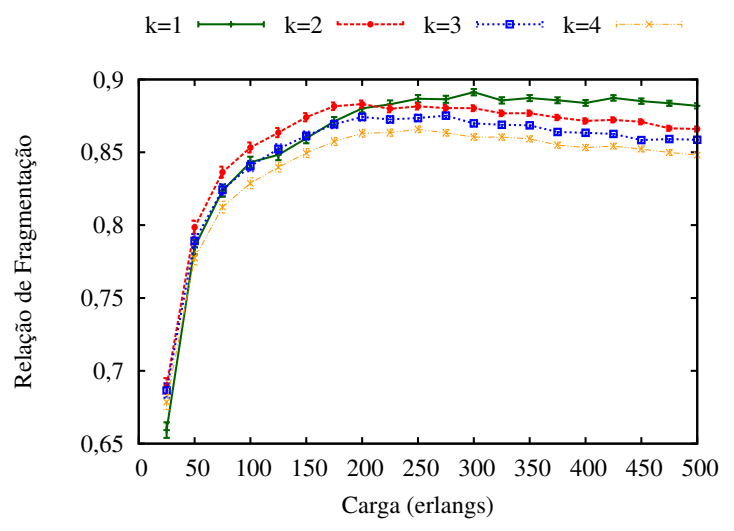

5.3 NSF

Figura 5. Relação de fragmentação em função da carga da rede

As Figuras 5.1, 5.2 e 5.3 mostram a relação de fragmentação gerada para as topologia Pan-European, USA e NSF. Para topologia USA (Figura 5.1), o algoritmo MPMN com caminho único produz a maior relação de fragmentação, o que explica a maior BBR gerada, isto acontece devido a conexão e desconexão de um maior número de slots. $\mathrm{O}$ algoritmo MPMN com $k=2$ produz quase $2 \%$ menos relação de fragmentação que o algoritmo MPMN com $k=1$, devido a melhor utilização de pequenos fragmentos de espectro quando utilizado roteamento multicaminho. Para $k=3$ e $k=4$, a diminuição da relação de fragmentação repete, apesar da relação de fragmentação reduzir menos de 1\%. A relação de fragmentação sofre maior influência da quantidade de múltiplos caminhos utilizados do que da BBR produzida.

Para a topologia USA (Figura 5.2), bem como para topologia Pan-European, o algoritmo MPMN com caminho único produz a maior relação de fragmentação, o que explica a maior BBR gerada. Diferentemente da topologia Pan-European, a relação de fragmentação dos diferentes valores de $k$ é mais próxima principalmente para caminhos únicos e múltiplos caminhos com $k=2$, evidenciando a influência das características da topologia USA.

Para a topologia NSF (Figura 5.3), sob cargas de até 200 erlangs, o algoritmo MPMN com $k=2$ produz a maior relação de fragmentação, o que explica a maior BBR gerada. A relação de fragmentação do algoritmo MPMN com caminho único sofre influência do menor número médio de saltos para os caminhos primário e de proteção, que por sua vez impacta na menor BBR gerada. A menor relação de fragmentação para $k=3 \mathrm{e}$ 
$k=4$ ocorre devido ao balanceamento da carga na rede gerada pelos diferentes caminhos. No entanto, o balanceamento da rede para $k=3$ e $k=4$ não é suficiente para produzir baixos valores de BBR, devido a baixa conectividade da rede.

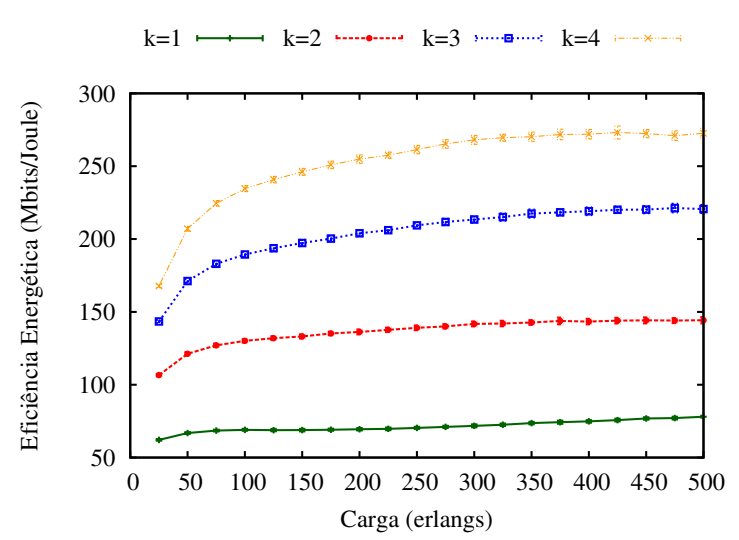

6.1 Pan-European

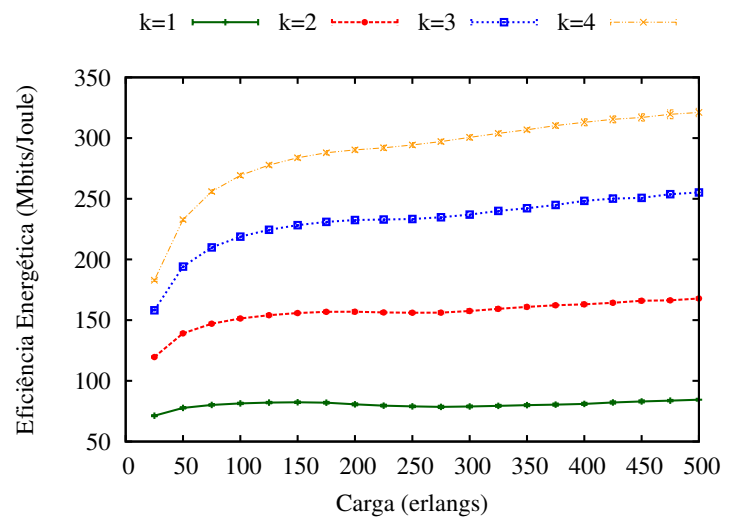

6.2 USA

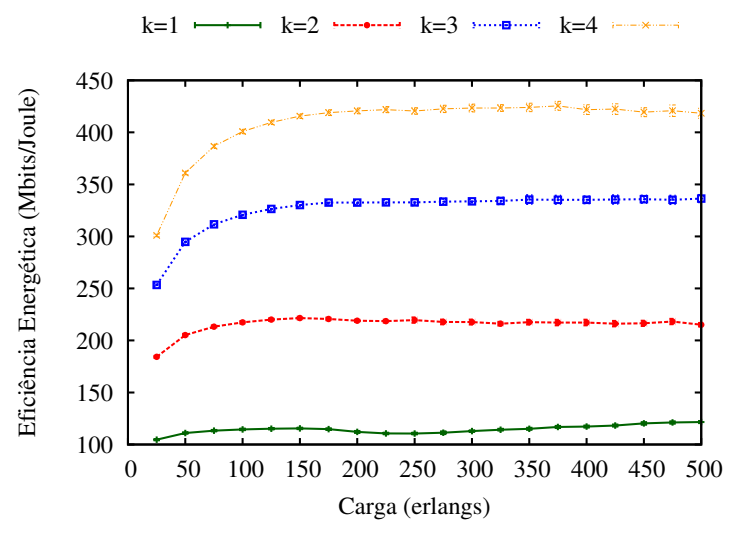

6.3 NSF

Figura 6. Eficiência energética em função da carga da rede

As Figuras 6.1, 6.2 e 6.3 mostram a eficiência energética gerada para as topologias Pan-European, USA e NSF. A comparação da eficiência energética em conexões que utilizam multicaminhos sofre influência do número médio de saltos dos caminhos, bem como, do consumo realizado pelo conjunto de slot do caminho. Por sua vez, o consumo do conjunto de slot do caminho varia de acordo com a demanda da requisição, bem como com o valor de $k$.

Para topologia Pan-European (Figura 6.1), com $k=4$, o algoritmo MPMN produz a maior eficiência energética, pois apesar deste algoritmo produzir o terceiro maior número médio de saltos do caminho primário, ele possui a menor requisição em número de slots por caminho. $\mathrm{O}$ algoritmo MPMN com $k=3$ produz a segunda maior eficiência energética, apesar de produzir número médio de saltos do caminho primário próximos ao produzido para $k=4$. Para $k=2$, produz-se a terceira menor eficiência energética mesmo utilizando-se o maior número médio de saltos dos caminhos primários. Para $k=1$, produz-se a menor eficiência energética, evidenciando o impacto entre o maior valor para $k$ e a eficiência energética.

Os resultados de eficiência energética gerados pelos diferentes valores de $k$ para a topologia USA (Figura 6.2) possuem comportamento similares aos resultados gerados para topologia Pan-European, isto acontece uma vez que a eficiência energética é influ- 
enciada pela divisão de caminhos. Conforme aumenta-se o número de caminhos para atender uma requisição, aumenta-se também a eficiência energética gerada.

Apesar do diferente comportamento na BBR gerada para topologia NSF em relação às outras topologias, a eficiência energética (Figura 6.3) gerada tem comportamento similar ao das outras topologias. Tal comportamento demonstra a grande influência da conectividade da topologia no consumo energético.

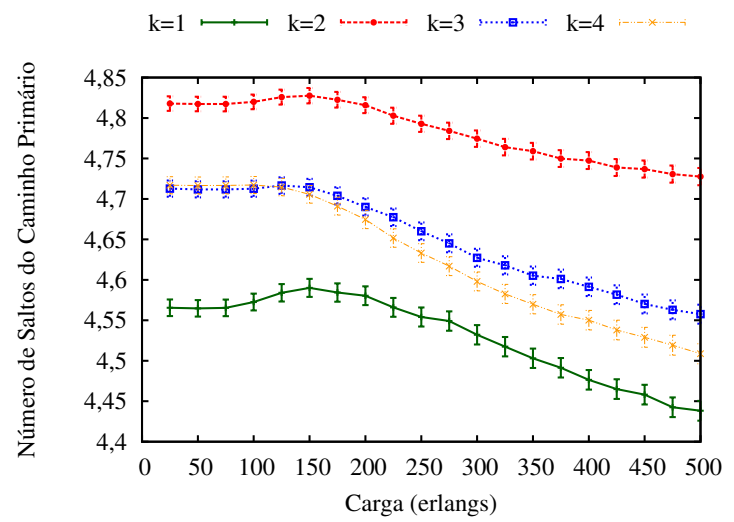

7.1 Pan-European

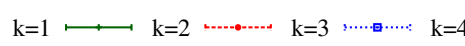

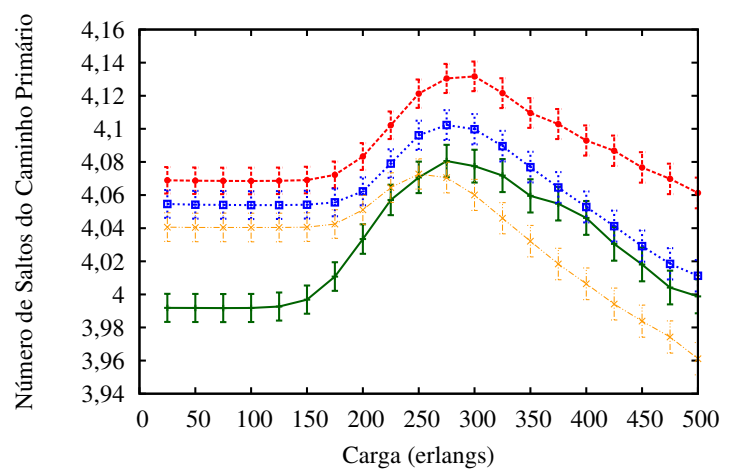

7.2 USA

$\mathrm{k}=1$ ط

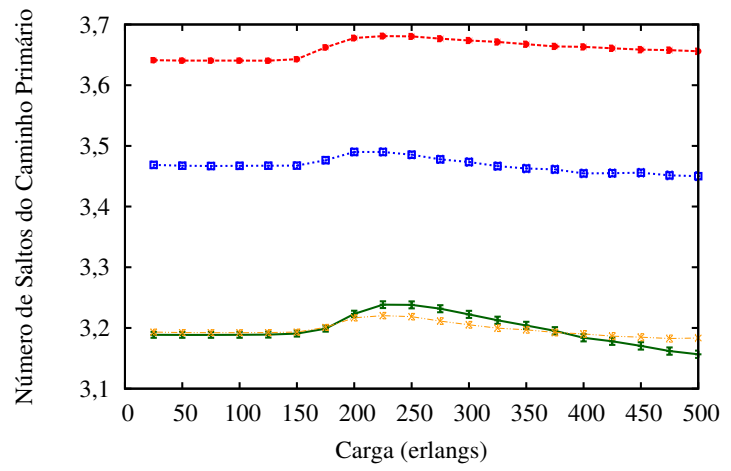

7.3 NSF

Figura 7. Média do número de saltos dos caminhos primários em função da carga da rede

As Figuras 7.1, 7.2 e 7.3 mostram a média do número de saltos dos caminhos primários gerados para as topologias Pan-European, USA e NSF. Para topologia PanEuropean (Figura 7.1), o algoritmo MPMN para $k=1$ produz o menor número médio de saltos dos caminhos primários já que só é necessário a criação de um caminho primário por requisição. Para $k=2$, o algoritmo MPMN produz a maior média do número médio de saltos dos caminhos primários; isto ocorre pois este algoritmo necessita da criação de dois caminhos primários por requisição, fazendo com que maiores caminhos sejam gerados. Para $k=3$ e $k=4$, o algoritmo MPMN produz número médio de saltos dos caminhos primários semelhantes.

Sob cargas baixas em que não existe bloqueio, a média do número médio de saltos dos caminhos primários permanece constante para topologia USA (Figura 7.2), como consequência da alta conectividade da topologia. Com o inicio da BBR em 150 erlangs para caminhos únicos e 175 erlangs para multicaminhos a média do número médio de saltos dos caminhos primários aumenta. Os maiores valores do número médio de saltos 
dos caminhos primários produzidos entre 250 e 300 erlangs explicam o maior bloqueio sob estas cargas.

Como nas topologias anteriores, para topologia NSF (Figura 7.3), o algoritmo MPMN mantém o número médio de saltos do caminho primário constante para cargas baixas em que não existe bloqueio. Com o inicio da BBR entre 150 e 225 erlangs o número médio de saltos do caminho primário aumenta, quando a BBR chega ao máximo, o número médio de saltos do caminho primário diminui. O pequeno aumento do número de saltos em relação às outras topologias acontece devido a baixa conectividade da topologia.

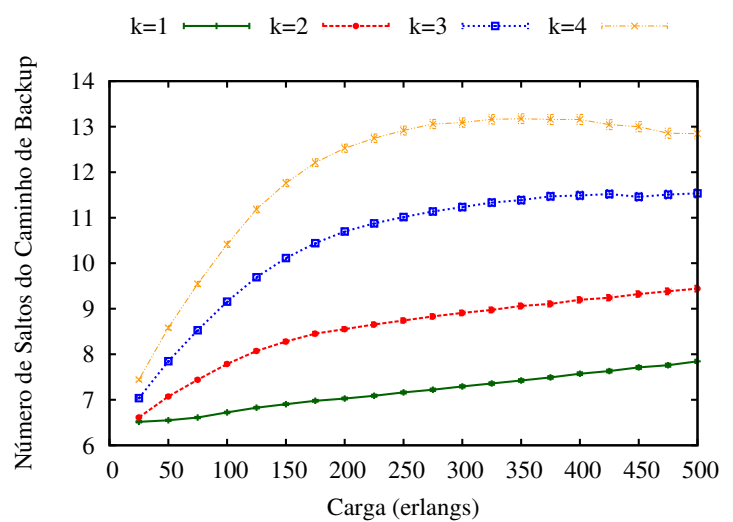

8.1 Pan-European

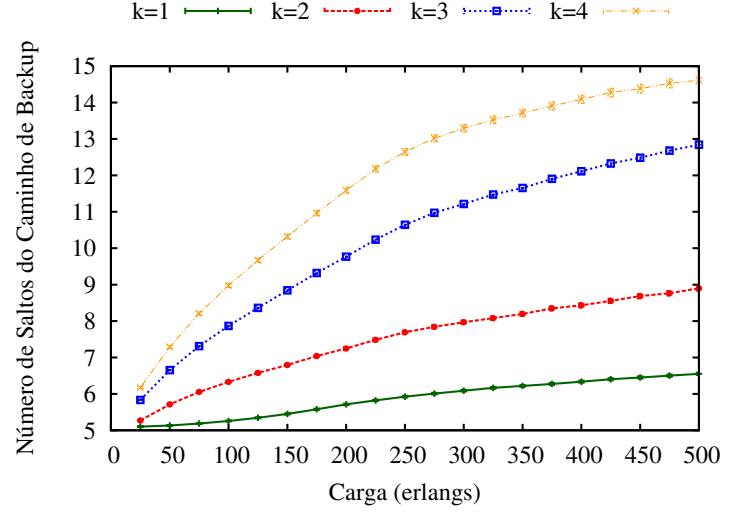

8.2 USA

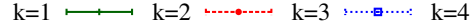

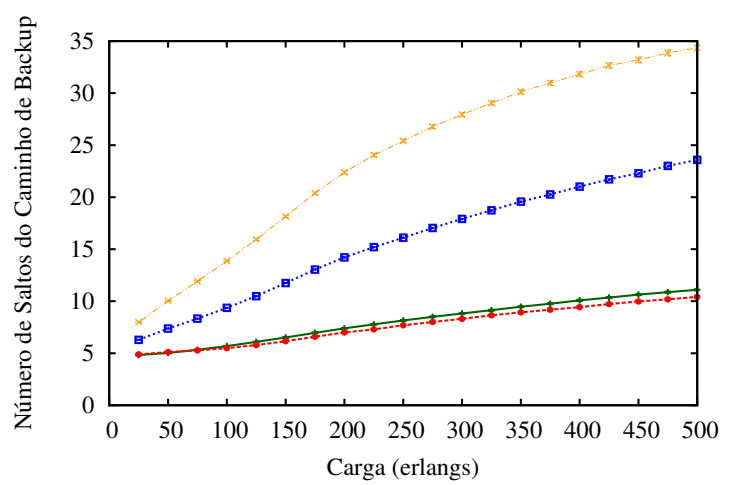

8.3 NSF

Figura 8. Média do número de saltos dos caminhos de proteção em função da carga da rede

As Figuras 8.1, 8.2 e 8.3 mostram o número médio de saltos dos caminhos de proteção gerados para as topologias Pan-European, USA e NSF.

Para topologia Pan-European (Figura 8.1), com o aumento do valor de $k$ para o algoritmo MPMN, é necessário um maior número de saltos do caminho de proteção pois os caminhos primários ocupam os menores caminhos e os caminhos de proteção devem ser disjuntos dos respectivos caminhos primários. Além disso, o número médio de saltos dos caminhos de proteção é sempre maior que o número médio de saltos dos caminhos primários.

Para topologia USA (Figura 8.2) bem como para a topologia Pan-European, o aumento do valor de $k$ implica no uso de um maior número de saltos do caminho de 
proteção, pois os caminhos primários ocupam os caminhos menores e os caminhos de proteção devem ser disjuntos dos respectivos caminhos primários. Além disso, o número médio de saltos dos caminhos de proteção é sempre maior que o número médio de saltos dos caminhos primários.

Para topologia NSF (Figura 8.3) diferentemente das outras topologias apresentadas em que com o aumento do valor $k$ para o algoritmo MPMN aumenta a média do número de saltos do caminho de proteção. Para topologia NSF, sob cargas baixas, a utilização de caminhos únicos produz número de saltos equivalente aos demandados por multicaminhos utilizando $k=2$. Além disso, sob cargas altas, a média do número de saltos do caminho de proteção é maior que a médio dos caminhos primários para $k=2$. Para $k=3$ e $k=4$, o comportamento é similar ao das outras topologias. O número médio de saltos do caminho de proteção chega ser dez vezes maior que o do número médio de saltos do caminho primário, principalmente para cargas altas.

\section{Conclusão}

Neste artigo, introduziu-se o algoritmo MPMN para proteção de redes ópticas elásticas com multiplexação por divisão espacial utilizando multicaminho. Comparou-se o uso deste algoritmo para diferentes números de multicaminhos. Os resultados mostram que apesar de algoritmos que utilizam maiores valor de $k$ demandarem um maior número de recursos, eles conseguem prover maior eficiência energética, devido ao balanceamento de recursos utilizados na rede, bem como, aos recursos reservados para o caminho de proteção que demandam baixo consumo de energia. A utilização de multicaminhos sofre grande influência da conectividade da topologia, pois as topologias com baixa conectividade dificultam a utilização de um número maior de caminhos entre o mesmo nó fonte e nó destino. A média do número médio de saltos do caminho de proteção pode ser 10 vezes maior que a média do número médio de saltos do caminho primário.

\section{Acknowledgments}

O presente trabalho foi realizado com apoio do Conselho Nacional de Desenvolvimento Científico e Tecnologico - Brasil (CNPq), processo número 151554/2018-8.

\section{Referências}

Chen, X., Chamania, M., Jukan, A., Drummond, A. C., and da Fonseca, N. L. S. (2009a). On the benefits of multipath routing for distributed data-intensive applications with high bandwidth requirements and multidomain reach. In 7th Annual Conference on Communication Networks and Services Research, CNSR 2009, 11-13 May 2009, Moncton, New Brunswick, Canada, pages 110-117.

Chen, X., Jukan, A., Drummond, A. C., and da Fonseca, N. L. S. (2009b). A multipath routing mechanism in optical networks with extremely high bandwidth requests. In Proceedings of the Global Communications Conference, 2009. GLOBECOM 2009, Honolulu, Hawaii, USA, 30 November - 4 December 2009, pages 1-6.

Chen, X., Zhong, Y., and Jukan, A. (2013). Multipath routing in elastic optical networks with distance-adaptive modulation formats. In 2013 IEEE International Conference on Communications (ICC), pages 3915-3920.

Consortium, T. (2002). A survey of multipath routing for traffic engineering. Technical report, Informations and Communications University.

de Santi, J. and da Fonseca, N. L. S. (2014). Energy-aware de-allocation of lightpaths in WDM networks. In IEEE Global Communications Conference, GLOBECOM 2014, Austin, TX, USA, December 8-12, 2014, pages 2586-2591. 
de Santi, J., Drummond, A. C., da Fonseca, N. L. S., and Chen, X. (2015). Holdingtime-aware dynamic traffic grooming algorithms based on multipath routing for WDM optical networks. Optical Switching and Networking, 16:21-35.

de Santi, J., Drummond, A. C., da Fonseca, N. L. S., Chen, X., and Jukan, A. (2012). Leveraging multipath routing and traffic grooming for an efficient load balancing in optical networks. In Proceedings of IEEE International Conference on Communications, ICC 2012, Ottawa, ON, Canada, June 10-15, 2012, pages 2989-2993.

Dharmaweera, M. N., Zhao, J., Yan, L., Karlsson, M., and Agrell, E. (2016). Trafficgrooming- and multipath-routing-enabled impairment-aware elastic optical networks. IEEE/OSA Journal of Optical Communications and Networking, 8(2):58-70.

Gao, T., Huang, S., Guo, B., Li, X., Kong, Q., Zhou, Y., Li, W., and Gu, W. (2016). Survivable multipath provisioning with content connectivity in elastic optical datacenter networks. In 2016 21 st OptoElectronics and Communications Conference (OECC) held jointly with 2016 International Conference on Photonics in Switching (PS), pages $1-3$.

Goścień, R., Walkowiak, K., and Tornatore, M. (2016). Survivable multipath routing of anycast and unicast traffic in elastic optical networks. IEEE/OSA Journal of Optical Communications and Networking, 8(6):343-355.

Hayashi, T., Taru, T., Shimakawa, O., Sasaki, T., and Sasaoka, E. (2011). Design and fabrication of ultra-low crosstalk and low-loss multi-core fiber. Opt. Express, 19(17):16576-16592.

Koshiba, M., Saitoh, K., Takenaga, K., and Matsuo, S. (2011). Multi-core fiber design and analysis: coupled-mode theory and coupled-power theory. Opt. Express, 19(26):B102B111.

Moura, P. M. and Drummond, A. C. FlexGridSim: Flexible Grid Optical Network Simulator. http://www.lrc.ic.unicamp.br/FlexGridSim/.

Muhammad, A., Zervas, G., Simeonidou, D., and Forchheimer, R. (2014). Routing, spectrum and core allocation in flexgrid sdm networks with multi-core fibers. In Proc. of ONDM Conf., pages 192-197, Stockholm, Sweden.

Oliveira, H. M. N. S. and da Fonseca, N. L. S. (2016). Algorithm for protection of space division multiplexing elastic optical networks. In 2016 IEEE Global Communications Conference (GLOBECOM), pages 1-6.

Ruan, L. and Zheng, Y. (2014). Dynamic survivable multipath routing and spectrum allocation in ofdm-based flexible optical networks. IEEE/OSA Journal of Optical Communications and Networking, 6(1):77-85.

Saridis, G. M., Alexandropoulos, D., Zervas, G., and Simeonidou, D. (2015). Survey and evaluation of space division multiplexing: From technologies to optical networks. IEEE Communications Surveys Tutorials, 17(4):2136-2156.

Tode, H. and Hirota, Y. (2014). Routing, spectrum and core assignment for space division multiplexing elastic optical networks. In Telecommunications Network Strategy and Planning Symposium (Networks), 2014 16th International, pages 1-7.

Yen, J. Y. (1970). An algorithm for finding shortest routes from all source nodes to a given destination in general networks. Quarterly of Applied Mathematics, 27(4):526-530. 